\title{
Are radiopharmaceuticals self-sterilizing? Radiation effect of gallium-68 and lutetium-177 on bacillus pumilus and staphylococcus succinus
}

\section{Simon Poetzsch}

Charite Universitatsmedizin Berlin - Campus Virchow-Klinikum

Winfried Brenner ( $\square$ winfried.brenner@charite.de)

https://orcid.org/0000-0003-2478-6004

\section{Sarah Spreckelmeyer}

Charite - Universitätsmedizin Berlin, corporate member of Freie Universität Berlin, Humboldt-Universität and Berlin Institute of Health, Department of Nuclear Medicine, Augustenburger Platz 1, 13353 Berlin https://orcid.org/0000-0003-1348-0309

Research article

Keywords: Radiopharmaceutical, sterilization, microorganism, aseptic

Posted Date: July 2nd, 2020

DOI: https://doi.org/10.21203/rs.3.rs-38980/v1

License: (9) (1) This work is licensed under a Creative Commons Attribution 4.0 International License. Read Full License 


\section{Abstract \\ Background}

For radiopharmaceuticals, aseptic preparation in combination with filtration is the most chosen sterilizing method. In general, the production of radiopharmaceuticals needs to fulfil the requirements of good manufacturing practice. In the scope of this work, we focused on the positron emitter gallium- 68 and on the therapeutically used beta- and gamma-emitter lutetium-177, as they are routinely used for in-house synthesis of radiopharmaceuticals in nuclear medicine departments. Our hypothesis is, that radiopharmaceuticals might be self-sterilizing due to a high radioactivity concentration and high-energy radionuclides in the preparation for intravenous injections.

\section{Results}

Incubation with gallium-68 and lutetium-177 for both 30 minutes and 5 hours post-dispensing did not cause any significant effect on bacteria growth. As the theoretical dose is only $0.1-0.6 \%$ of the Ph. Eur. recommended dose of $25 \mathrm{kGy}$, we conclude that the beta and positron energy of lutetium-177 and gallium-68 as used for standard radiopharmaceutical in-house production is not sufficient to decrease the number of colony forming units compared to the control values.

\section{Conclusions}

Based on these findings, gallium-68 and lutetium-177 labeled radiopharmaceuticals are not selfsterilizing under the tested conditions with respect to bacillus pumilus and staphylococcus succinus. Consequently, strict aseptic preparation conditions in addition to end-sterilization of the radiopharmaceutical e.g. through membrane filtration are strongly advised for in-house productions.

\section{Background:}

Radiopharmaceuticals are injections or infusions for diagnostic or therapeutic use which effect is caused by the incorporated radionuclide. Radioactivity has, depending on its character, a very high energy that can cause single- or even double-strand breaks of DNA that eventually lead to apoptosis of the respective cells.[1] In nuclear medicine, this feature is used to detect or even treat various diseases. As radiopharmaceuticals are applied parenterally, they must be sterilized before use. In the European Pharmacopoeia, six different methods are described to achieve sterility with a sterility assurance level (SAL) of $10^{-6}$ - steam sterilization, dry heat, radiation, gas, membrane filtration and working under aseptic conditions (Ph. Eur. Vol 9.0, 5.1.1). For radiopharmaceuticals, aseptic preparation in combination with membrane filtration is the most chosen sterilizing method. Other methods are usually not applicable due to the short half-lives of the radionuclides and/or incompatibility of the radiopharmaceutical with elevated temperatures as they may contain heat-sensitive biomolecules. In general, the production of 
radiopharmaceuticals needs to fulfil the requirements of good manufacturing practice (GMP) and sterile (starting) materials are used wherever possible. The (automated) production is performed under aseptic conditions in laminar-air flow hoods class A or in hot cells/ isolators using synthesis modules in GMP clean rooms. The final step of the production is mostly the filtration of the radiopharmaceutical through a $0.22 \mu \mathrm{m}$ membrane filter for end-sterilization. In order to check for sterility conformity, the radiopharmaceutical is incubated (retrospectively) for two weeks on growth media as described in the European Pharmacopoeia (Ph. Eur. Vol 9.0, 2.6.1).

Our hypothesis is, that radiopharmaceuticals might be self-sterilizing due to the fact that they contain a high radioactivity concentration and high-energy radionuclides. To the best of our knowledge, this hypothesis was thus far only tested for $\left[{ }^{99 \mathrm{~m}} \mathrm{Tc}\right]$-radiopharmaceuticals $[2,3]$ and $\left[{ }^{18} \mathrm{~F}\right]-$ radiopharmaceuticals[4]. Brown et al. state, that the time lag between preparation and the sterility test of $\left[{ }^{99 \mathrm{~m}} \mathrm{Tc}\right]$-radiopharmaceuticals should be as short as possible. Their reasoning is, that longer time lags have a greater chance to obtain negative sterility results although the preparation might have been already contaminated at the time of dispensing. Jörg et al. investigated the "autosterilization" effect of $\left[{ }^{18} \mathrm{~F}\right]$-radiopharmaceuticals and concluded, that intrinsic $\left[{ }^{18} \mathrm{~F}\right]$-radiation is not sufficient for achieving sterility of the radiopharmaceutical. In our opinion, the main drawbacks, which we will avoid in our experiment set-up of these studies, were the long incubation times of up to 11 hours with the radiation source and the direct inoculation of the radiopharmaceutical of interest with the microorganisms. These long incubation times are not realistic in the daily routine because in-house synthesized radiopharmaceuticals in nuclear medicine departments are usually applied within one hour after preparation to the patients. Furthermore, samples taken directly out of the inoculated microorganismsradiopharmaceutical solution contain remaining radioactivity. Thus, the seeded sample on growth media may be effected by the remaining radioactivity.

In the scope of this work, we will focus on the positron emitter gallium-68 and on the therapeutically used beta- and gamma-emitter lutetium-177, as they are routinely used for in-house synthesis of radiopharmaceuticals in nuclear medicine departments. Lutetium- 177 is primarily a beta-emitter (490 keV) that decays after 6.7 days to the stable hafnium- 177 but also emits gamma rays with an energy of $113 \mathrm{keV}(3 \%)$ and $210 \mathrm{keV}(11 \%)$. Gallium-68 is a positron emitter (1899 keV (88\%) and $822 \mathrm{keV}$ (1\%)) with gamma energies of $511 \mathrm{keV}(178 \%)$ and $1077 \mathrm{keV} \mathrm{(3 \% )} \mathrm{with} \mathrm{a} \mathrm{half-life} \mathrm{of} 68$ minutes.[5] Both radionuclides can be linked to peptides such as DOTATOC (edotreotide) and PSMA.[6]

The growth behavior of two different germs have been evaluated at two different steps during the preparation - either pre-dispensing or post-dispensing. Bacillus pumilus is a known radiation resistant species and can be used to validate ionizing radiation sterilization as used in the European Pharmacopoeia as sterility marker.[7] Additionally, this germ is resistant to environmental stresses. Staphylococcus succinus was chosen as a member of the wide-spread genus staphylococcus.[8] Both germs are Gram-positive and categorized in the lowest biological safety class and can be handled in normal laboratories. 
As gallium-68 and lutetium-177 labeled radiopharmaceuticals are mostly prepared in-house, we assumed that the time of injection will be within 30 minutes post-dispensing. Consequently, we chose 30 minutes as first incubation time point. We also added a 5 hours incubation point post-dispensing, as this might be relevant for lutetium-177 radiopharmaceuticals when they are produced ahead of injection.

Additionally, pre-dispensing measures were chosen. During the synthesis of e.g. $\left[{ }^{68} \mathrm{Ga}\right] \mathrm{Ga}$-DOTATOC, the starting materials are heated to $95^{\circ} \mathrm{C}$ for approximately $15 \mathrm{~min}$. Here, we tested the effect of an elevated temperature on the bacteria after 15 minutes. Additionally, gallium-68 radiopharmaceuticals are often post-processed with a maximum of $10 \%(\mathrm{~V} / \mathrm{V})$ ethanol. To take this also into account, we tested the bacterial growth after 30 min incubation with $10 \%(\mathrm{~V} / \mathrm{V})$ ethanol.

\section{Results}

\section{Staphylococcus succinus}

The post-dispensing results of the 30 minutes and 5 hours incubations are shown in Fig. 1. After 30 minutes incubation, the amount of colony forming units (cfu) in the control fraction increases to approximately double the amount compared to the start value. The gallium- 68 and lutetium- 177 treated samples show a three-fold increase of cfu after 30 minutes of incubation at room temperature in $0.9 \%$ $\mathrm{NaCl}$ solution. The increases in cfu are not significant compared to control values ( $p=0.44$ for gallium-68 and $p=0.42$ for lutetium-177).

After 5 hours of incubation, the control and radiation treated samples show no significant change in cfu $(p=0.45$ for gallium-68 and $p=0.77$ for lutetium-177).

\section{Bacillus Pumilus}

The results of the 30 minutes and 5 hours incubation experiment post-dispensing are shown in Fig. 3 . After 30 minutes, the amount of cfu in the control fraction does not change compared to the start value. The gallium-68 and lutetium-177 treated samples show no significant change compared to the control values ( $p=0.30$ for gallium- 68 and $p=0.83$ for lutetium-177) of cfu after 30 minutes of incubation at room temperature in $0.9 \% \mathrm{NaCl}$ solution.

After 5 hours of incubation, the gallium- 68 treated samples show a trend towards a decrease in growth ( $p$ $=0.17$ ), although without statistical significance. The lutetium- 177 treated samples show no significant change in growth compared to the control value $(p=0.89)$.

\section{Theoretical Dose}

The theoretical dose was calculated using standard radiochemical formulas (Supporting Information). The theoretically achievable dose in the radiopharmaceuticals produced in-house ranges from 45 Gy to 
$161 \mathrm{~Gy}$ for gallium-68 and $12 \mathrm{~Gy}$ to $119 \mathrm{~Gy}$ for lutetium-177. Compared to the radiation dose to achieve an SAL of $10^{\wedge}-6$, we achieve only $0.1-0.6 \%$ of the necessary dose of $25 \mathrm{kGy}$ (Table 1 ).

Table 1

Overview of theoretical dose in radiopharmaceuticals.

\begin{tabular}{|c|c|c|c|c|}
\hline Nuclide & $\begin{array}{l}\text { Dose concentration } \\
\text { [GBq/mL] }\end{array}$ & $\begin{array}{l}\text { Incubation } \\
\text { time }\end{array}$ & $\begin{array}{l}\text { Theoretical dose } \\
\text { [Gray] }\end{array}$ & $\begin{array}{l}\text { Ratio to } 25 \text { kGy Ph. } \\
\text { Eur. [\%] }\end{array}$ \\
\hline $\begin{array}{l}\text { Gallium- } \\
68\end{array}$ & 0.2 * & $30 \mathrm{~min}$ & 44.54 & 0.2 \\
\hline $\begin{array}{l}\text { Gallium- } \\
68\end{array}$ & $0.2^{*}$ & $5 \mathrm{~h}$ & 161.09 & 0.6 \\
\hline $\begin{array}{l}\text { Lutetium- } \\
177\end{array}$ & $0.1^{\star \star}$ & $30 \mathrm{~min}$ & 11.98 & 0.1 \\
\hline $\begin{array}{l}\text { Lutetium- } \\
177\end{array}$ & $0.1^{\star *}$ & $5 \mathrm{~h}$ & 118.59 & 0.5 \\
\hline \multicolumn{5}{|c|}{ *Ga-68 preparations in in-house productions: $2 \mathrm{GBq}$ in $10 \mathrm{~mL}$} \\
\hline \multicolumn{5}{|c|}{ **Lu-177 preparations in in-house productions: $7 \mathrm{GBq}$ in $80 \mathrm{~mL}$} \\
\hline
\end{tabular}

\section{Discussion}

Within 30 minutes and 5 hours of incubation, the gallium- 68 and lutetium- 177 treated samples show no significant decrease of colony forming units. As the theoretical dose is only $0.1-0.6 \%$ of the Ph. Eur. recommended dose of $25 \mathrm{kGy}$, we conclude that the beta and positron energy of lutetium-177 and gallium-68 are not sufficient to decrease the number of cfu compared to the control value. Sterility can thus not be achieved through self-sterilization by the chosen radionuclides.

The pre-dispensing experiments however show, that elevated temperature at $95^{\circ} \mathrm{C}$ for 15 min leads to a complete destruction of the tested viable microorganisms. The addition of $10 \%(\mathrm{~V} / \mathrm{V})$ ethanol decreases the cfu to $27 \%$ and $83 \%$ for staphylococcus succinus and bacillus pumilus, respectively. Bacillus pumilus is a spore-forming germ. Several bacillus strains e.g. bacillus pumilus were tested towards their hardiness to ethanol by P. Thomas. He came to the conclusion, that bacillus pumilus is resistant to ethanol up to a concentration of about aqueous 90\% (V/V) ethanol.[9] Elzain et. al describe the antibacterial effect of different concentrations of ethanol on various staphylococcus species. The authors reported, that staphylococcus species are sensitive to ethanol and ethanol can thus be used as an antimicrobial.[10] Our results match those results described in the literature. Although these results may be promising in regards to achieving sterility with elevated temperatures during the preparation step, the radiopharmaceutical could still get contaminated during the subsequent dispensing step.

\section{Conclusions:}


Based on these findings, gallium-68 and lutetium-177 labeled radiopharmaceuticals are not selfsterilizing under the tested in-house production conditions with respect to bacillus pumilus and staphylococcus succinus. Consequently, strict aseptic preparation conditions in addition to endsterilization of the radiopharmaceutical e.g. through membrane filtration are strongly advised for in-house productions.

\section{Material And Methods}

All materials used were sterile single use materials e.g. syringes. Gallium-68 was eluted from the pharmaceutical grade ${ }^{68} \mathrm{Ge} /{ }^{68} \mathrm{Ga}$ generator (GalliaPharm, Eckert \& Ziegler Radiopharma $\mathrm{GmbH}$, Germany). Lutetium-177 was obtained from ITG Garching. Bacillus pumilus (DSM 492) and Staphylococcus succinus (DSM 105508) were obtained from DSMZ-German Collection of Microorganisms and Cell Cultures $\mathrm{GmbH}$. Radioactivity counting was performed using a borehole counter (Nuklear-Medizintechnik Dresden $\mathrm{GmbH}$, Germany). Cultivation media tryptone soya broth USP was obtained from OXOID Deutschland $\mathrm{GmbH}$. Bacteria concentration was calculated with plate count agar (146269) bought from Merck KGaA. All other chemicals were obtained from Sigma Aldrich.

\section{Cultivation Of Bacteria}

Bacillus pumilus and staphylococcus succinus are stored at $-80^{\circ} \mathrm{C}$ in tryptone soya broth USP with $25 \%$ (V/V) glycerol as cryoprotectant. For every experiment, an overnight culture was established from the frozen culture. For that, a spatula tip of frozen bacteria culture was transferred with a sterile pipette tip into a sterile $15 \mathrm{~mL}$ falcon tube filled with $14 \mathrm{~mL} \mathrm{NaCl}$ solution. After homogenizing the bacteria solution, $50 \mu \mathrm{L}$ was transferred into $10 \mathrm{~mL}$ cultivation media for overnight culture at $37^{\circ} \mathrm{C}$.

\section{Post-dispensing Experiments}

The overnight culture was homogenized. The bacteria vials for the control and gallium-68/lutetium-177 treatment were prepared as follows: One $\mathrm{mL}$ (approximately $1 \times 10^{\wedge} 9 \mathrm{cfu}$ ) of the overnight culture was added to $9 \mathrm{~mL} 0.9 \% \mathrm{NaCl}$ solution and homogenized. A sample from this solution was taken to estimate the cfu at the beginning of the experiment (start). As control values, samples were taken from the untreated bacteria-solution after 30 minutes and 5 hours (control).

A $1.5 \mathrm{~mL}$ glas vial containing either $1 \mathrm{~mL}$ gallium-68 (approx. 1-2 GBq) or $1 \mathrm{~mL}$ lutetium-177 (approx. $0.8-1.4 \mathrm{GBq}$ ) was immersed into the $0.9 \% \mathrm{NaCl}$ bacteria vial for 30 min or 5 hours. Before taking samples at 30 min or 5 hours, the bacteria vial was homogenized.

$100 \mu \mathrm{l}$ of the bacteria vial was used for further dilutions and seeding on counting agar plates. After $24 \mathrm{~h}$, the cfu were counted and the total cfu calculated as described below. 


\section{Pre-dispensing Experiments}

The overnight culture was homogenized. The bacteria vials for the ethanol and $95^{\circ} \mathrm{C}$ treatment were prepared as follows: One $\mathrm{mL}$ (approximately $1 \times 10^{\wedge} 9 \mathrm{cfu}$ ) of the overnight culture was added to $9 \mathrm{~mL}$ $0.9 \% \mathrm{NaCl}$ solution and homogenized in case of the $95^{\circ} \mathrm{C}$ treatment. For the ethanol treatment, $1 \mathrm{~mL}$ sterile ethanol was added to $8 \mathrm{~mL} 0.9 \% \mathrm{NaCl}$ solution and one $\mathrm{mL}$ of bacteria were added.

$100 \mu \mathrm{l}$ of the bacteria vial was used for further dilutions and seeding on counting agar plates. After $24 \mathrm{~h}$, the cfu were counted and the total cfu calculated as described below.

\section{Calculation Of Total cfu}

For calculation of the total cfu, a dilution series of the bacteria vial was taken into account.

\begin{tabular}{|llll|}
\hline Dilution & name & Volume of Solution from bacteria vial $[\mathrm{mL}]$ & Volume $0.9 \% \mathrm{NaCl}[\mathrm{mL}]$ \\
\hline 1 & A1 & & \\
\hline $1: 100$ & A2 & $0.1 \mathrm{~mL}$ of $\mathrm{A} 1$ & 9.9 \\
\hline $1: 1000$ & A3 & $1 \mathrm{~mL}$ of $\mathrm{A} 2$ & 9 \\
\hline
\end{tabular}

Overall the number of bacteria in the bacteria vial after the incubation time can be calculated by number of colonies * dilution * 100 .

\section{Abbreviations}

SAL

sterility assurance level

cfu

colony forming units

\section{Declarations}

\section{Ethics approval and consent to participate}

Not applicable

Consent for publication

Not applicable.

Availability of data and materials 
All data generated or analyzed during this study are included in this published article and its supplementary information files.

\section{Competing interests}

The authors declare that they have no competing interests.

\section{Funding}

Not applicable.

\section{Authors' contributions}

SP designed, performed and analyzed the experiments. WB evaluated the results concerning clinical applications. SS designed, performed and analyzed the experiments and repeated the experiments in order to proof its repeatability. All authors contributed to, read and approved the final manuscript.

\section{Acknowledgement}

Not applicable.

\section{References}

1. Barnard S, Bouffler S, Rothkamm K. The shape of the radiation dose response for DNA double-strand break induction and repair. Genome Integr. 2013;4(1):1.

2. Brown S, Baker MH. The sterility testing of dispensed radiopharmaceuticals. Nucl Med Commun. 1986;7(5):327-36.

3. Stathis VJ, et al. Effect of technetium Tc $99 \mathrm{~m}$ pertechnetate on bacterial survival in solution. Am J Hosp Pharm. 1983;40(4):634-7.

4. Jorg G, et al. Inhibition of viability of microorganisms in [(18)F]-labeled radiopharmaceuticals. Nucl Med Biol. 2017;44:105-13.

5. [cited 2020 9th June 2020]; Available from: http://www.nucleide.org/Laraweb/index.php.

6. Breeman WA, et al. (68)Ga-labeled DOTA-peptides and (68)Ga-labeled radiopharmaceuticals for positron emission tomography: current status of research, clinical applications, and future perspectives. Semin Nucl Med. 2011;41(4):314-21.

7. Prince HN. D-values of Bacillus pumilus spores on irradiated devices (inoculated product). Appl Environ Microbiol. 1978;36(2):392-3.

8. Place RB, et al. Staphylococcus succinus subsp. casei subsp. nov., a dominant isolate from a surface ripened cheese. Syst Appl Microbiol. 2002;25(3):353-9. 
9. Thomas P. Long-term survival of Bacillus spores in alcohol and identification of $90 \%$ ethanol as relatively more spori/bactericidal. Curr Microbiol. 2012;64(2):130-9.

10. Abdelrhman M, Elzain SME. Mohammed Elfatih Ahmed Ibrahim, Effectiveness of ethanol and methanol alcohols on different isolates of staphylococcus species. Journal of Bacteriology \& Mycology, 2019. 7(4).

\section{Figures}

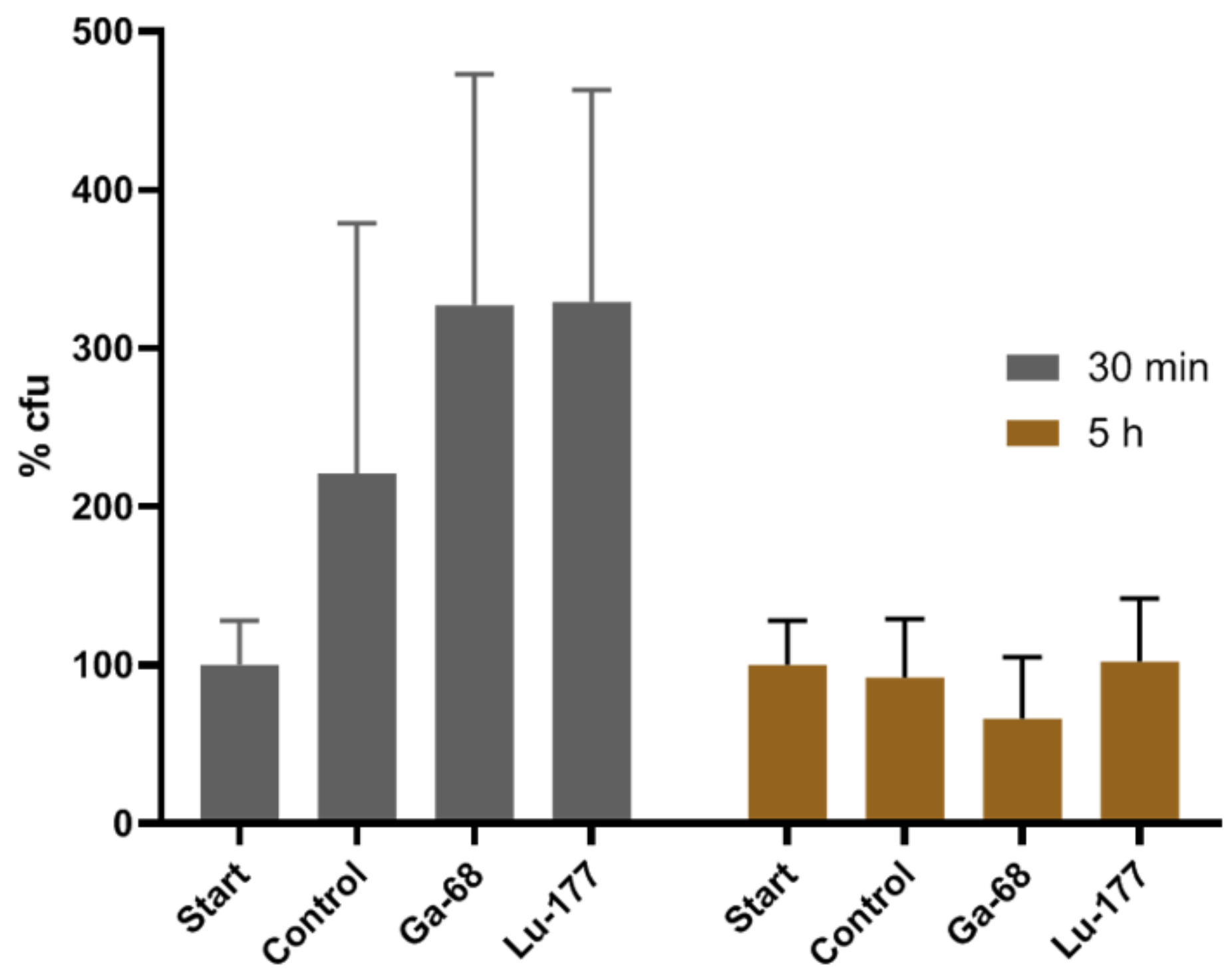

Figure 1

Staphylococcus succinus: Results of 30 min incubation and 5 hours incubation; $n \geq 3$ 


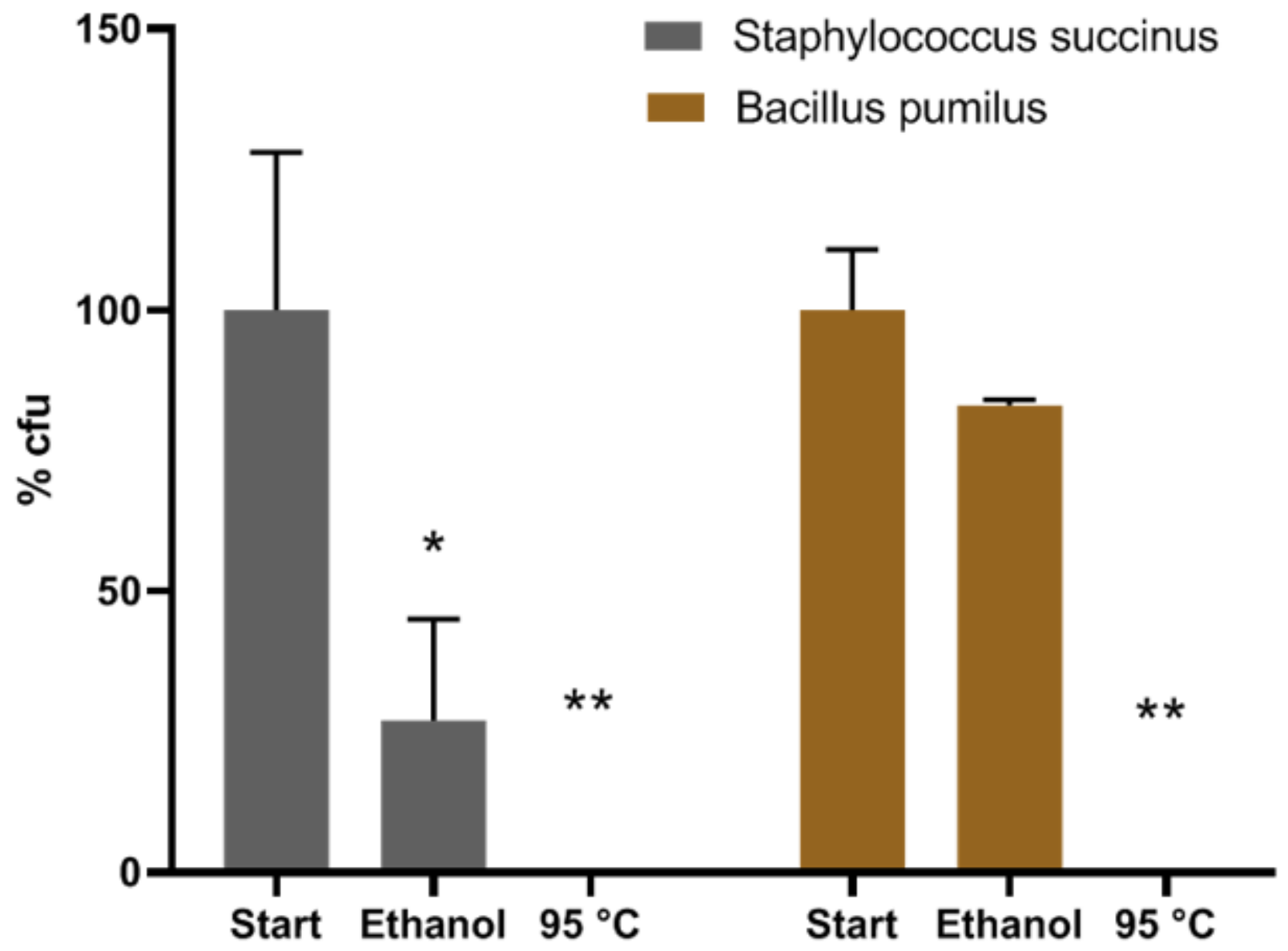

Figure 2

Results of incubation with ethanol or at $95^{\circ} \mathrm{C}$ of the two different bacteria species; $\mathrm{n} \geq 3$ ( ${ }^{*}=p$-value $<0.05$ $\star *$ p-value $<0.005)$ 


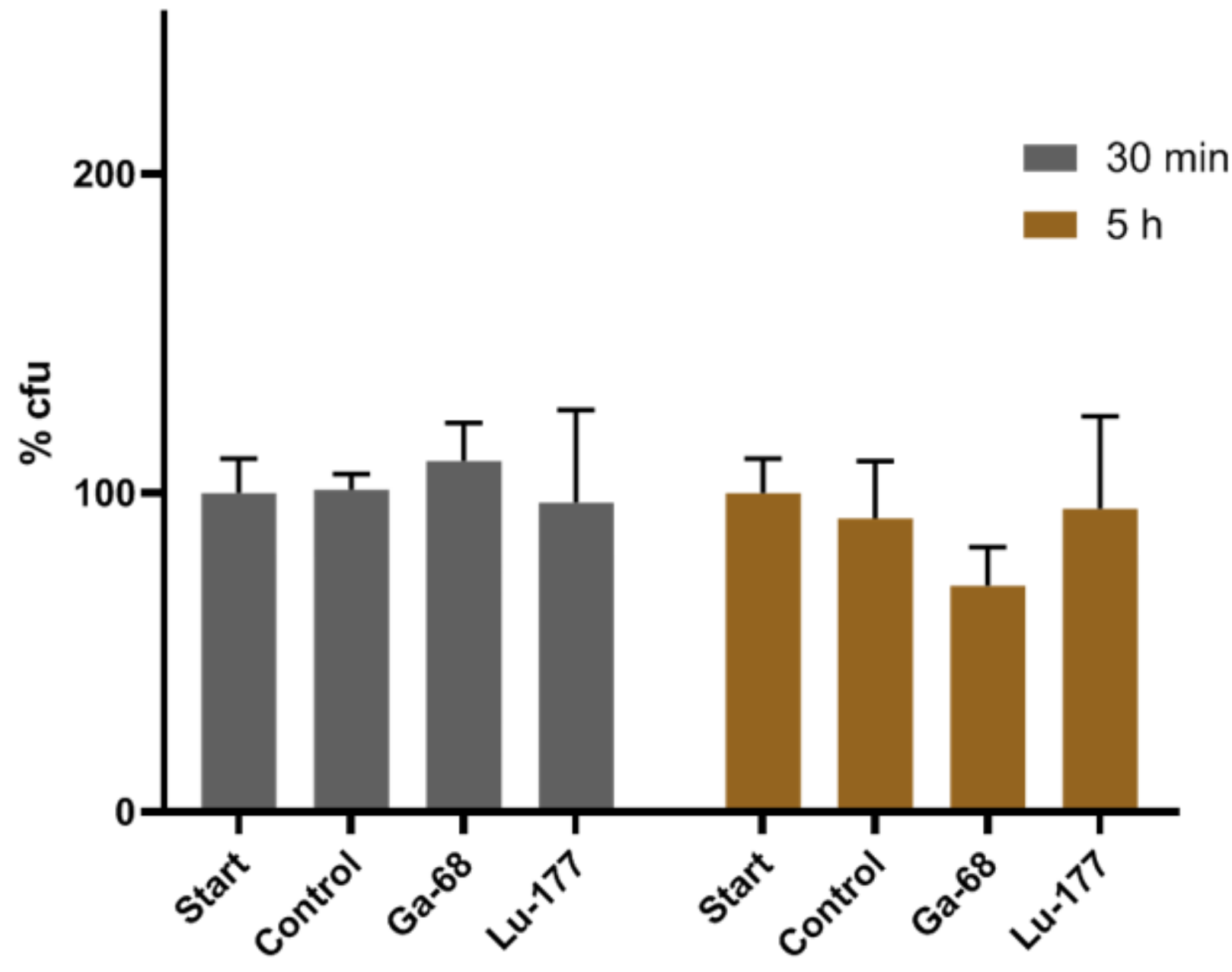

Figure 3

Bacillus pumilus: Results of 30 min incubation and 5 hours incubation; $n \geq 3$

\section{Supplementary Files}

This is a list of supplementary files associated with this preprint. Click to download.

- PoetzschBrennerSpreckelmeyerSupportingInformation.docx 